\title{
Plane electromagnetic wave propagation of pulsed periodic field in conductive half-space
}

\author{
Viktor Timofeev, Eduard Vinter, Anatolii Gulyashinov \\ Electrical Equipment and Electrical Process Technology Department, Siberian Federal \\ University, Krasnoyarsk, Russian Federation \\ evinter@sfu-kras.ru
}

Viktor Timofeev was qualified as an Engineer in specialty of "Power Plants" in Polytechnical Institute (Krasnoyarsk) in 1974. He received the degree of the Candidate of Technical Sciences in 1994. In 1994, he was awarded the degree of the Doctor of Technical Sciences. He is currently a Professor with the Electrical Equipment and Electrical Process Technology Department, Polytechnical Institute of Siberian Federal University, Krasnoyarsk, Russia. He has authored or coauthored over 100 papers in journals and conferences. His current research interest includes research into electrical process equipment for metallurgical purposes.

Eduard Vinter received a master's degree in Electrical Engineering in Metallurgy at the Siberian Federal University (Krasnoyarsk) in 2016. Ltd. Since 2018, he is assistant of the Electrical Equipment and Eletrical Process Technology Department in the Polytechnic Institute of the Siberian Federal University. His current research interest includes mathematical modeling of electromagnetic, thermal and hydrodynamic processes in electrical technology equipment of metallurgical purpose.

Anatolii Gulyashinov received a master's degree in Electrical Engineering in Metallurgy at the Siberian Federal University (Krasnoyarsk) in 2016. Since 2018, he is assistant of the Electrical Equipment and Eletrical Process Technology Department in the Polytechnic Institute of the Siberian Federal University. His current research interest includes the electromagnetic field analysis in complex electrical equipment for metallurgy. 
Plane electromagnetic wave propagation of pulsed periodic field in conductive half-space

Nowadays, application of pulsed periodic fields in various technological devices is of interest, in particular for the influencing on a conductive media. In this paper solution of the problem of the propagation of a plane electromagnetic wave of pulsed periodic field created by a planar inductor, in the conductive half-space is presented. The solution is obtained in the form of Fourier series as a function of a complex argument. The differential and integral characteristics of the "inductorconductive half-space" system for different values of the inductor time constant are determined. A comparative analysis of the obtained results with the electromagnetic characteristics of plane sinusoidal electromagnetic wave propagation process in conductive half-space is made.

Keywords: pulsed periodic electromagnetic field, electromagnetic field, plane electromagnetic wave, Fourier series

\section{Introduction}

The application of the effect of alternating electromagnetic field on a conductive body is widespread in modern industry [1]. A widely used technology of the electromagnetic field influence on a conductive body is induction heating by which operations of hardening, welding, soldering, and also melting of metals are performed [2, 3]. Also, the metallurgical industry widely applies the technology of electromagnetic stirring of liquid metals $[4,5,6]$. The purpose of this is to temperature equalization and chemical composition homogenization throughout the volume of a furnace. In general, these installations can be characterized as electromagnetic devices for influencing the conductive medium in order to implement a specific technological process. Despite the fact that the technological purpose of the device may be different, the principles of conversion of electrical energy are common to these devices.

As a rule, a sinusoidal voltage of low, industrial or high frequency is used as the power supply of the mentioned installations $[2,7]$. However, at present, due to the 
development of power converter technology, it is of interest to use non-sinusoidal periodic voltage as a power supply of electromagnetic devices for influence on a conductive body. The form of a non-sinusoidal voltage can be unipolar rectangular pulses, alternating rectangular pulses, triangular pulses, or a sequence of sinusoidal voltage pulses.

In [8], study of the effectiveness of pulsed voltage in an induction hardening unit has been carried out. The authors have shown that the heating process using a pulsed voltage in the millisecond range is characterized by a high power density in the workpiece, as well as the possibility of quenching without the use of an external quenching means.

In $[9,10,11]$, numerical and experimental studies of the heat transfer of a conductive fluid in a low-frequency pulsed electromagnetic field have been carried out. The possibility of increasing the intensity of heat transfer and increasing the turbulent kinetic energy of the flow due to the impact of pulsed electromagnetic force have been shown.

In [12], numerical simulation and experimental study of an electromagnetic stirrer which is supplied by a pulsed periodic current has been carried out. This paper reports that with certain parameters and operating modes of an electromagnetic stirrer with pulsed periodic currents, more efficient stirring of liquid metals is performed. Decrease in reactive power consumption and duration of stirring is also noted.

In $[13,14]$, a numerical and experimental study of the melt stirring efficiency using the pulse sequences of a rotating magnetic field has been carried out. Paper has reported that this stirring method provides equiaxed microstructure without macrosegregation. 
Thus, the some advantages of using pulsed periodic voltage in electromagnetic devices to influence the conductive medium have been shown. However, at the same time, a theoretical study of the general regularities of electromagnetic processes and distribution pattern of the electromagnetic field in a conductive body was not carried out. To study the distribution pattern of the electromagnetic field and the power density in a conductive body, it is rational to consider the problem of the plane electromagnetic wave propagation, in which the vectors $\mathrm{E}$ and $\mathrm{H}$ vary according to a pulsed periodic law. This wave case is valid for many types of induction devices with a plane or cylindrical configuration.

In this paper, problem of plane electromagnetic wave propagation in a conductive half-space of a pulsed periodic field created by a planar inductor is solved. The solution is obtained in the form of a Fourier series in a complex form. The instantaneous values of the E-field and $\mathrm{H}$-field vectors, the distribution of electromagnetic power over the depth of the conductive half-space, as well as the value of the active power density on the surface of the half-space are obtained. This paper is arranged as follows: the problem statement is described in Section 2. Section 3 presents the solution of differential equations and the definition of the Pointing vector. Results and discussion are presented in Section 4.

\section{Problem statement}

First, it should be noted that the case of propagation in a conductive half-space of a plane electromagnetic wave, in which the vectors $\mathrm{E}$ and $\mathrm{H}$ vary in a sinusoidal law, is described in detail in the course of theoretical electrical engineering [15].

Electromagnetic characteristics for this case are compared with the characteristics obtained in this paper. Consider the case of a plane electromagnetic wave propagation of a pulsed field in a homogeneous electrically conductive medium with a specific 
conductivity $\gamma$ and magnetic permeability $\mu_{0}$ (non-magnetic material). Conductive medium has infinity dimensions. Center of the rectangular coordinate system is located on the surface of half-space so that the $\mathrm{x}$ axis coincides with the direction of the $\mathrm{H}$ vector, and the $\mathrm{z}$ axis coincides with the direction of the $\mathrm{E}$ vector. $\mathrm{Y}$ axis is directed deep into the conductive space. Thus $\mathrm{H}$ and $\mathrm{E}$ vectors in the design model comprise as follows:

$$
\boldsymbol{H}=\boldsymbol{e}_{\boldsymbol{x}} H_{x} ; \boldsymbol{E}=\boldsymbol{e}_{z} E_{z},
$$

where $\boldsymbol{e}_{\boldsymbol{x}}$ и $\boldsymbol{e}_{z}$ - unit vectors.

Such a plane electromagnetic wave can be generated by a plane inductor located near unbounded conductive space (Figure 1) [16]. The inductor is a system of rectilinear conductors of unlimited length along the $\mathrm{z}$ coordinate. The number of conductors along the $\mathrm{x}$ coordinate is also unlimited.

Figure 1. Plane inductor located near unbounded conductive space

If there are $W$ conductors at the length $l$ and electric current $i(t)$ flows in each conductor, then the $\mathrm{H}$-field in the space between the inductor and the conductive body is determined by the expression

$$
H(t)=j(t)=\frac{W \cdot i(t)}{l}
$$

where $j(t)$ - linear current density of the inductor winding, $\mathrm{A} \mathrm{m}^{-1}$

Suppose that the inductor winding has an active resistance $R$ and inductance $L$, and is connected to a source of rectangular voltage $u(t)$ with magnitude $U$ (Fig. 2). Then the linear current density of the inductor vary according to the function 


$$
j(t)= \begin{cases}J_{m}-2 J_{m} e^{-t / \tau} ; & 0 \leq t<T / 2, \\ -J_{m}+2 J_{m} e^{-(t / \tau+T / 2)} ; & T / 2 \leq t<T .\end{cases}
$$

where $\tau=L / R$-inductor time constant, s.

Figure 2. Pulsed periodic voltage and linear current density

\section{Theory}

\subsection{Electromagnetic field equation and boundary conditions}

The H-field in the region of the conductive half-space the following equation and boundary conditions are correct (the indexes are omitted hereinafter)

$$
\begin{gathered}
\frac{\partial^{2} H(t, y)}{\partial y^{2}}-\mu_{0} \gamma \frac{\partial H(t, y)}{\partial t}=0, \\
H(\infty, t)=0 ; \quad \mathrm{H}(0, t)=j(t) .
\end{gathered}
$$

\subsection{Solving electromagnetic field equation}

Let us find a solution to equation (4) in the form of Fourier series in a complex form [17].

$$
H(y, t)=\sum_{n=-\infty}^{n=+\infty} H_{n}(y) e^{i k_{n} t}
$$

Here

$$
\begin{gathered}
H_{n}(y)=\frac{1}{T} \int_{0}^{\mathrm{T}} H_{n}(y, t) e^{-i k_{n} t} \partial t, \\
k_{n}=\frac{2 n \pi}{T} .
\end{gathered}
$$


Having transposed (4) in compliance with (7) and (8) the following results are obtained:

$$
\frac{\mathrm{d}^{2} H_{n}(y)}{\mathrm{d} y^{2}}-\beta_{n}{ }^{2} H_{n}(y)=0 .
$$

Here

$$
\beta_{\mathrm{n}}=\sqrt{i k_{n} \mu_{0} \gamma}=\frac{\sqrt{n}}{\delta}(i+1)=\frac{\sqrt{2 n}}{\delta} e^{i \pi / 4},
$$

where $\delta$-penetration depth of the first harmonic of electromagnetic wave.

Equation (10) is an ordinary differential equation of the second order, the general solution of which is written as follows

$$
H_{n}(y)=C_{1} e^{\beta_{n} y}+C_{2} e^{-\beta_{n} y}
$$

where $C_{1}, C_{2}$-integration constants determined from boundary conditions.

Boundary conditions (5) and (6) shall also be transposed in line with (7) and (8).

$$
H_{n}(0)=\Psi_{n}=I_{n 1}+I_{n 2} ; H_{n}(\infty)=0
$$

Here

$$
\begin{gathered}
I_{n 1}=J_{m}\left[\frac{i}{2 n \pi}\left(e^{-i n \pi}-1\right)+\frac{2 e^{T / 2 \tau}}{(T / \tau+i 2 n \pi)}\left(e^{-(T / \tau+i 2 n \pi)}-1\right)\right], \\
I_{n 2}=-J_{m}\left[\frac{i}{2 n \pi}\left(e^{-i 2 n \pi}-e^{-i n \pi}\right)+\frac{2 e^{T / 2 \tau}}{(T / \tau+i 2 n \pi)}\left(e^{-(T / \tau+i 2 n \pi)}-e^{-(T / 2 \tau+i 2 n \pi)}\right)\right] .
\end{gathered}
$$

Defining the integration constants and put them in (12), we obtain the solution 


$$
H(y, t)=\sum_{n=-\infty}^{n=+\infty} \Psi_{n} e^{-\beta_{n} y} e^{i k_{n} t}
$$

From the Maxwell equation, the E-field is determined

$$
E(y, t)=-\frac{1}{\gamma} \frac{\partial H(y, t)}{\partial y}=\frac{1}{\gamma} \sum_{n=-\infty}^{n=+\infty} \beta_{n} \Psi_{n} \mathrm{e}^{-\beta_{n} y} \mathrm{e}^{i k_{n} t}
$$

Applying the Euler formula to expressions (17) and (18) and taking the real part, we write the expressions for the instantaneous values of the magnetic and electric fields

$$
\begin{gathered}
H(\hat{y}, \hat{t})=2 J_{m} \sum_{n=1}^{n=\infty}\left|\hat{\Psi}_{n}\right| e^{-\sqrt{n} \hat{y}} \cos \left(\alpha_{n}-\sqrt{n} \hat{y}+2 n \pi \hat{t}\right), \\
E(\hat{y}, \hat{t})=\frac{2 J_{m}}{\gamma \delta} \sum_{n=1}^{n=\infty} \sqrt{2 n}\left|\hat{\Psi}_{n}\right| \mathrm{e}^{-\sqrt{n} \hat{y}} \cos \left(\alpha_{n}-\sqrt{n} \hat{y}+2 n \pi \hat{t}+\pi / 4\right) .
\end{gathered}
$$

where $\hat{\Psi}_{n}=\Psi_{n} / J_{m} ; \hat{y}=y / \delta ; \hat{t}=t / T ;\left|\hat{\Psi}_{n}\right|, \alpha_{n}$ - absolute value and argument of complex number $\hat{\Psi}_{n}$.

\subsection{Pointing vector}

In compliance with (6), the instantaneous value of the Poynting vector on the surface of the conductive half-space

$$
\Pi(0, t)=[E(0, t) \cdot H(0, t)]=\frac{2 J_{m}^{2}}{\gamma \delta} \sum_{n=1}^{\infty} \sqrt{2 n}\left|\hat{\Psi}_{n}\right| \cos \left(k_{n} t+\alpha_{n}+\pi / 4\right) \cdot \hat{j}(t)
$$

The average value of the Poynting vector for the period on the surface of the conductive half-space corresponds to the active electromagnetic power transmitted to the conductive space through the unit surface, $\mathrm{W} \mathrm{m} \mathrm{m}^{-2}$. 


$$
P_{e m}=\frac{2 J_{m}^{2}}{\gamma \delta} \sum_{n=1}^{\infty} \sqrt{2 n}\left|\hat{\Psi}_{n}\right| \cdot N_{n}
$$

Here

$$
N_{n}=\frac{1}{T} \int_{0}^{T}\left[\cos \left(k_{n} t+\alpha_{n}+\pi / 4\right) \cdot \hat{j}(t)\right] \partial t
$$

\section{Results and discussion}

For convenience of analysis, let us turn to relative quantities, dividing the obtained expressions for the H-field (19), E-field (20), and the power (24) by their base values

$$
H_{b}=J_{m} ; \quad E_{b}=\frac{J_{m}}{\gamma \delta} ; \quad P_{b}=\frac{J_{m}^{2}}{2 \gamma \delta}
$$

In compliance with (15), (16), (19) and (20), the electromagnetic characteristics depend on the inductor time constant or on unitless parameter $\hat{\tau}=\tau / T$. It should be noted that at the moments of rise and droop of the H-field, the E-field, and, consequently, the power, tend to infinity. Moreover, the Gibbs phenomenon occurs for small $\hat{\tau}$, which complicates the calculation and analysis. Guided by these factors, as well as on the basis of practical considerations, the range of study $\hat{\tau}$ chosen from 0.01 to 0.2 .

In figure 3 instantaneous relative $\mathrm{H}$-field (a) and E-field (b) over the period at different depths of the conductive space for $\hat{\tau}=0.05$ are shown. At the depth $\hat{y} \succ 1$, the higher harmonics appear slightly, and the shape of the H-field and E-field is almost sinusoidal. It follows from (19) and (20), such filtering of higher harmonics over the depth of a half-space is explained by the fact that the degree of damping of a higher harmonic is proportional to the root of its number $\sqrt{n}$. 
Figure 3. (a) Relative instantaneous H-field and (b) E-field, over the period

Figure 4 shows the distribution of the relative surface power density over depth of half-space for various $\hat{\tau}$. The dashed line corresponds to the distribution for the case of plane wave of sinusoidal field. It can be seen that in the case of a pulsed periodic field the distribution of the relative active power density over depth has more sudden damping pattern. At the same time, for a wide range of time constant, power density distribution over depth has higher values of the, compared with a sinusoidal field.

Figure 4. Distribution of the relative surface power density

Figure 5 shows the relative surface power density on the surface of the conductive halfspace $(\hat{y}=0)$ versus $\hat{\tau}$. The dashed line corresponds to the relative value of the surface power density for the case of a plane wave of a sinusoidal field. For values $\hat{\tau} \prec 0.138$ the active power density on the surface of the half-space is higher than for the case of a sinusoidal field. For the minimum investigated value of the time constant $(\hat{\tau}=0.01)$, the active power density on the surface of the conductive half-space is about 2.3 times greater than a sinusoidal field. Based on the above mentioned, it can be concluded that reducing inductor time constant is equivalent to increasing the frequency of the sinusoidal electromagnetic field.

Figure 5. Relative surface power density versus relative inductor time constant

Figure 6 shows the active power fraction released in the layer of first harmonic penetration depth $(\hat{y}=1)$ versus relative time constant $\hat{\tau}$. The dashed line corresponds the value of power released in the layer $\delta$ in the case of a plane wave of a sinusoidal field (86.5\% of the total active power). It follows from the figure 6 that the closer the shape of the $\mathrm{H}$-field is to the rectangular, the more active power fraction is released in the layer $\delta$ (up to $90 \%$ of the total active power). With an increase of the inductor time constant, the value of the active power fraction in the layer $\delta$ decreases and at values $\hat{\tau} \succ 0.15$ ceases to change. At the same time, in the entire investigated range of $\hat{\tau}$, in the 
$\delta$ layer a greater amount of active power is released, as compared with the plane wave of the sinusoidal field.

Figure 6. Active power fraction released in the layer of first harmonic penetration depth versus relative inductor time constant

\section{Conclusion}

Compared to the plane electromagnetic wave of sinusoidal field for pulsed periodic field characterized by:

(1) Increase the power density on the surface of the half-space with decreasing inductor time constant, that is equivalent to increasing the frequency of the sinusoidal field;

(2) A larger value of the active power released in the layer of the first harmonic penetration depth;

(3) Filtration of higher harmonics by the depth of the conductive half-space.

The interest of further research is the study of the spatial and time-frequency distributions of electromagnetic forces in a conductive body under the influence of a pulsed periodic field.

References:

1. Nacke B. Theoretical Background and Aspects of Electrotechnologies: Physical Principles and Realization: Intensive Course. Basic I. Publishing house of ETU; 2012.

2. Rudnev V, Loveless D, Cook R. Handbook of induction heating. Second edition. ed. Boca Raton, FL: CRC Press, Taylor \& Francis Group; 2017. (Manufacturing engineering and materials processing; 61).

3. Rudnev V. Induction Heating and Heat Treating for Aerospace Applications. Adv Mater Process. 2018 Feb-Mar;176(2):58-61. PubMed PMID: WOS:000426999300018; English. 
4. Fdhila R., Sand U., Eriksson JE, et al. A. stirring history. ABB Review. 2016;3:45-48.

5. Timofeev V. Control of convective flows in liquid metal in fluenced by electromagnetic forces. Engineering \& Technologies. 2015;5(1):28-38.

6. Timofeev V., Khatsayuk M. Theoretical Design Fundamentals for Mhd Stirrers for Molten Metals. Magnetohydrodynamics. 2016 Oct-Dec;52(4):495-506. PubMed PMID: WOS:000394414000006; English.

7. Grundas $\mathrm{S}$. Advances in induction and microwave heating of mineral and organic materials Preface. Advances in Induction and Microwave Heating of Mineral and Organic Materials. 2011:IX-xii. doi: Book_Doi 10.5772/562. PubMed PMID: WOS:000386947600001; English.

8. Aliferov A., Forzan M., Lupi S. Milliseconds pulse induction hardening. International Journal of Microstructure and Materials Properties. 2018;13(12):73-84.

9. Musaeva D, Ilin V, Baake E, et al. Numerical Simulation of the Melt Flow in an Induction Crucible Furnace Driven by a Lorentz Force Pulsed at Low Frequency. Magnetohydrodynamics. 2015 Oct-Dec;51(4):771-783. PubMed PMID: WOS:000369117200010; English.

10. Musaeva D., Baake E, Ilin V. Experimental Investigation of Al-Alloy Directional Solidification in Pulsed Electromagnetic Field2016.

11. Musaeva D, Baake E, Ilin V, et al. Analysis of the Almgsi-Alloy Structure Formed under the Influence of Low-Frequency Pulsed Lorentz Force. Magnetohydrodynamics. 2017 Apr-Jun;53(2):245-254. PubMed PMID: WOS:000406079700003; English.

12. Timofeev V., Lybzikov G., Khatsayuk M., et al. Magnetohydrodynamic stirrer liquid metal with a non-sinusoidal currents. Engineering \& Technologies. 2015;6(2):166-177.

13. Eckert S, Nikrityuk P., Rabiger D., et al. Efficient melt stirring using pulse sequences of a rotating magnetic field: Part I. Flow field in a liquid metal column. Metall Mater Trans B. 2007 Dec;38(6):977-988. doi: 10.1007/s11663007-9096-4. PubMed PMID: WOS:000251925700015; English.

14. Willers B., Eckert S., Nikrityuk P., et al. Efficient melt stirring using pulse sequences of a rotating magnetic field: Part II. Application to solidification of Al-Si alloys. Metall Mater Trans B. 2008 Apr;39(2):304-316. doi: 
10.1007/s11663-008-9126-x. PubMed PMID: WOS:000255879400014; English.

15. Cheng D.. Field and wave electromagnetics. Reading, Mass.: Addison-Wesley; 1989. (The Addison-Wesley series in electrical engineering).

16. Lupi S., Forzan M., Aliferov A. Induction and direct resistance heating. Springer; 2015.

17. Zill D, Wright W., Cullen M.. Advanced engineering mathematics. Jones \& Bartlett Learning; 2011.

This work was supported by the [Russian Foundation for Basic Research, Government of Krasnoyarsk Territory, Krasnoyarsk Regional Fund of Science] under Grant [\#18-48242013: "Investigation of the effect of spatial and frequency-time distributions of electromagnetic forces in the melt on the character of MHD processes in the liquid metal"]. 